\title{
FINITE COVERINGS BY SUBGROUPS WITH A GIVEN PROPERTY
}

\author{
by MARC A. BRODIE and LUISE-CHARLOTTE KAPPE
}

(Received 15 November, 1991)

1. Introduction. Let \& be a group-theoretic property. We say a group has a finite covering by $\mathbb{E}$-subgroups if it is the set-theoretic union of finitely many $\mathbb{E}$-subgroups. The topic of this paper is the investigation of groups having a finite covering by nilpotent subgroups, $n$-abelian subgroups or 2-central subgroups.

R. Baer $[12 ; 4.16]$ characterized central-by-finite groups as those groups having a finite covering by abelian subgroups. In [6] it was shown that $\left[G: Z_{c}(G)\right]$ finite implies the existence of a finite covering by subgroups of nilpotency class $c$, i.e. $\mathfrak{N}_{c}$-groups. However, an example of a group is given there which has a finite covering by $\mathfrak{N}_{2}$-groups, but $Z_{2}(G)$ does not have finite index in the group. These results raise two questions, on which we will focus our investigations.

1. Can one characterize groups with $\left[G: Z_{c}(G)\right]$ finite in terms of certain finite coverings by subgroups of nilpotency class $c$ ?

2. For a given property \&, can one find a characteristic subgroup $E$ of $G$ such that $G$ has a finite covering by $E$-subgroups if and only if $E$ has finite index in $G$ ?

Several other characterizations of central-by-finite groups can be given. A very interesting one is due to B. H. Neumann in [11]: A group is central-by-finite if and only if every subset of pairwise noncommuting elements is finite. Two other characterizations can be easily given: A group is central-by-finite if and only if it has only finitely many maximal abelian subgroups, and if and only if every maximal abelian subgroup has finite index in the group. Theorem 2.1 summarizes these characterizations. Theorem 2.2 contains the affirmative answer to our first question together with the analogues of the other characterizations given in Theorem 2.1. A slightly stronger result can be given for 2-Engel groups. However, a 2-Engel group can have a finite covering by $\mathfrak{R}_{2}$-subgroups even if the second center has infinite index in the group (Theorem 2.4).

Turning to our second question, Baer's result shows that for \& abelian the center is the desired characteristic subgroup. In [6], the question was answered for the property 2-Engel, denoted by $\mathbb{E}_{2}$. It was established that

$$
L(G)=\{a \in G \mid[a, x, x]=1 \forall x \in G\},
$$

the subgroup of right 2-Engel elements, has finite index in $G$ if and only if $G$ has a finite covering by $\mathbb{E}_{2}$-subgroups.

In Sections 3 and 4 we investigate the properties $n$-abelian, 2-central and nilpotency of class 2 in the context of the second question. In all four cases where there is an affirmative answer, the characteristic subgroups are what have been called E-embedded normal subgroups for the respective properties [5, [4]. There, A. Dietrich discusses E5-embedded normal subgroups in the context of finite groups with emphasis on properties for which there exists a unique maximal $\mathbb{E}$-embedded normal subgroup. In reference to [4], we make the following definition:

Definition 1.1. Let $\&$ be a group-theoretic property. A subgroup $H$ of $G$ is called Glasgow Math. J. 35 (1993) 179-188. 
Es-embedded if $\langle H, x\rangle \in \mathbb{E}$ for all $x$ in $G$. A property $\&$ is called rigid if there exists a unique maximal $\mathbb{E}$-embedded normal subgroup for every group $G$. This unique maximal subgroup will be called the rigid E-kernel of $G$, denoted by $K(G, \mathbb{E})$.

The margin of a variety $\mathfrak{B}$ is always a $\mathfrak{B}$-embedded normal subgroup, but it is not necessarily maximal. It can easily be seen that the existence of an 5 -embedded normal subgroup of finite index is equivalent to the existence of a finite $\mathfrak{5}$-covering of the group (Proposition 3.1). Such normal subgroups need not be characteristic in the group, as we will see in the case of the property $\mathfrak{N}_{2}$ (Theorem 4.3 ).

For easier reference, we conclude this introduction with a quote of B. H. Neumann's Lemma, which is basic for our investigations.

THEOREM 1.2. ([10;4.4]) Let $G=\bigcup_{i=1}^{n} H_{i} g_{i}$ where $H_{1}, \ldots, H_{n}$ are (not necessarily distinct) subgroups of $G$. Then if we omit from the union any coset $H_{i} g_{i}$ for which $\left[G: H_{i}\right]$ is infinite, the union of the remaining cosets is still all of $G$.

2. Groups with $Z_{c}(G)$ of finite index. In this section we characterize groups in which a term of the upper central series has finite index in the group, analogous to the characterizations given for central-by-finite groups in the following theorem:

THEOREM 2.1. For a group $G$ the following conditions are equivalent:

(i) $G$ is central-by-finite;

(ii) $G=\bigcup_{i=1}^{k} H_{i}$, where $H_{i}^{\prime}=1$;

(iii) any subset $S$ of pairwise noncommuting elements of $G$ is finite;

(iv) $G$ has only finitely many maximal abelian subgroups;

(v) every maximal abelian subgroup has finite index in $G$.

Proof. The equivalence of (i), (ii) and (iii) is due to R. Baer $[12 ; 4.16]$ and B. H. Neumann [11]. Obviously (i) implies (iv) and (v). Next, note that every $x \in G$ is contained in a maximal abelian subgroup of $G$. Assume (iv). Then $G=\bigcup_{i=1}^{k} M_{i}$, where $M_{i}$ is a maximal abelian subgroup of $G$. Thus (iv) implies (ii). Finally, assume (v). Let $x \in M$, a maximal abelian subgroup. Then $M \subseteq C_{G}(x)$. Since $[G: M]<\infty$, it follows $\left[G: C_{G}(x)\right]<$ $\infty$. Thus $G$ is an $F C$-group. By Lemma 2 in [11] it follows that $G$ is central-by-finite. Hence (v) implies (i).

A group having a finite covering by $\Re_{2}$-subgroups does not necessarily have $Z_{2}(G)$ of finite index [6]. However, the existence of finite coverings by $\mathfrak{R}_{c}$-subgroups which are specially embedded in the group, is equivalent to having $Z_{c}(G)$ of finite index. Consider the following set of subgroups of a group $G$ :

$$
\mathscr{H}(G, c)=\left\{H \leq G ; H^{\prime} \subseteq Z_{c-1}(G)\right\} .
$$

Obviously, $H \in \mathscr{H}(G, c)$ implies $H \in \mathfrak{R}_{c}$. One can easily see that $\mathscr{H}(G, c)$ has maximal elements. We denote this subset of $\mathscr{H}(G, c)$ by $\mathscr{M}(G, c)$. Since $Z_{0}(G)=1$, we see that the 
elements in $\mathcal{M}(G, 1)$ coincide with the maximal abelian subgroups of the group. If $c>1$, the maximal $\mathfrak{N}_{c}$-subgroups are not necessarily contained in $\mathcal{M}(G, c)$.

The following theorem characterizes groups with $Z_{c}(G)$ of finite index. The case $c=1$ is exactly the statement of Theorem 2.1 .

THEOREM 2.2. For a group $G$ and a positive integer $c$ the following are equivalent:

(i) $G / Z_{c}(G)$ is finite;

(ii) $G=\bigcup_{i=1}^{k} H_{i}, H_{i} \in \mathscr{H}(G, c)$;

(iii) any subset $S$ of $G$, where $[x, y] \notin Z_{c-1}(G)$ for all distinct $x, y \in S$, is finite;

(iv) $\mathcal{M}(G, c)$ is finite;

(v) the elements of $\mathcal{M}(G, c)$ have finite index in $G$.

For the proof of Theorem 2.2 and later results we need a lemma in context with marginal subgroups. P. Hall in [5] introduced the margin $\psi^{*}(G)$ of a word $\psi\left(x_{1}, \ldots, x_{n}\right)$. For the definition of a partial margin and the margin $\psi_{V}^{*}(G)$ of a variety $\mathfrak{B}$ defined by the set of laws $V$, see [6].

LEMma 2.3. Let $G$ be a group and $H$ a subgroup of $G$. In addition, let $\mathfrak{B}$ be a variety and $\phi_{V}^{*}(G)$ the marginal subgroup of $\mathfrak{B}$ in $G$. If $H \in \mathfrak{B}$, then $H \phi_{V}^{*}(G) \in \mathfrak{B}$. If $G / \phi_{V}^{*}(G)$ is finite, then $G$ has only finitely many maximal $\mathfrak{B}$-subgroups, each of finite index.

Proof. This is an immediate consequence of the definition of a marginal subgroup.

Proof of Theorem 2.2. Let $\tilde{G}=G / Z_{c-1}(G)$. We will show that $\tilde{G}$ satisfies (2.1.n) for $\mathrm{n}=\mathrm{i}, \ldots, \mathrm{v}$ of Theorem 2.1 if and only if $G$ satisfies the corresponding condition (2.2.n) of Theorem 2.2. Since (2.1.i) through (2.1.v) are equivalent by Theorem 2.1, it will follow that (2.2.i) through (2.2.v) are equivalent.

Now $[\tilde{G}: Z(\tilde{G})]=\left[G / Z_{c-1}(G): Z_{c}(G) / Z_{c-1}(G)\right]=\left[G: Z_{c}(G)\right]$. Thus (2.1.i) holds for $\bar{G}$ if and only if (2.2.i) holds for $G$.

Let $H=\mathscr{H}(G, c), h_{1}, h_{2} \in H, x_{1}, x_{2} \in Z_{c}(G), g_{1}, \ldots, g_{c-1} \in G$. Consider $H Z_{c}(G)$. Then $\left[x_{1} h_{1}, x_{2} h_{2}, g_{1}, \ldots, g_{c-1}\right]=\left[h_{1}, h_{2}, g_{1}, \ldots, g_{c-1}\right]=1$, since $Z_{c}(G)$ is the margin of the variety $\mathfrak{l}_{c}$ and $H^{\prime} \subseteq Z_{c-1}(G)$. Thus $\left(H Z_{c}(G)\right)^{\prime} \subseteq Z_{c-1}(G)$, and hence $H Z_{c}(G) \in$ $\mathscr{H}(G, c)$.

Assume (2.2.ii), i.e. $G=\bigcup_{i=1}^{k} H_{i}, H_{i} \in \mathscr{H}(G, c)$. Since $Z_{c-1}(G) \subseteq Z_{c}(G)$ it follows by the above that $H_{i} Z_{c-1}(G) \in \mathscr{H}(G, c)$, and hence $G=\bigcup_{i=1}^{k} H_{i} Z_{c-1}(G)$. So $\tilde{G}=\bigcup_{i=1}^{k} \tilde{H}_{i}$, where $\tilde{H}_{i}=H_{i} Z_{c-1}(G) / Z_{c-1}(G)$, and hence (2.2.ii) for $G$ implies (2.1.ii) for $\tilde{G}$. Similarly, (2.1.ii) for $\tilde{G}$ implies (2.2.ii) for $G$.

Suppose there exists an infinite set $\tilde{S}$ of pairwise noncommuting elements in $\tilde{G}$. This is equivalent to the existence of an infinite set $S$ of elements in $G$ such that $[a, b] \notin Z_{c-1}(G)$ for distinct $a, b \in S$. Hence $\tilde{G}$ satisfies (2.1.iii) if and only if $G$ satisfies (2.2.iii).

To prove the equivalence of the last two statements, we observe that there is a one-one correspondence between the elements in $\mathcal{M}(G, c)$ and the maximal abelian subgroups of $\tilde{G}$. Thus it follows that $\mathcal{M}(\tilde{G}, 1)$ is finite if and only if $\mathcal{M}(G, c)$ is finite, and hence (2.1.iv) holds in $\tilde{G}$ if and only if (2.2.iv) holds in $G$. 
Observing that for $M \in M(G, c)$ and $M / Z_{c-1}(G)=\tilde{M} \in \mathcal{M}(\tilde{G}, 1)$, we have

$$
[\tilde{G}: \tilde{M}]=\left[G / Z_{c-1}(G): M / Z_{c-1}(G)\right]=[G: M],
$$

we obtain that (2.1.v) is satisfied in $\tilde{G}$ if and only if (2.2.v) is satisfied in $G$.

For 2-Engel groups we can prove a stronger result. The existence of only finitely many maximal $\mathfrak{N}_{2}$-subgroups is equivalent to $\left[G: Z_{2}(G)\right]<\infty$. However, the existence of a finite $\mathfrak{N}_{2}$-covering alone does not imply that $Z_{2}(G)$ has finite index even in the case of an $\mathscr{F}_{2}$-group. The following lemma is used in the proof of our theorem:

Lemma 2.4. Let $G$ be a 2-Engel group. Then $C_{G}\left(G^{\prime}\right)=Z_{2}(G)$.

Proof. It follows from the Jacobi identity that for any group $Z_{2}(G) \subseteq C_{G}\left(G^{\prime}\right)$. If $G \in \mathcal{F}_{2}$ and $a, x, y \in G$, then $[a, x, y]^{3}=1$ and $[a, x, y]^{2}=[a,[x, y]]$. If $a \in C_{G}\left(G^{\prime}\right)$, then $[a, x, y]=1$. Hence $C_{G}\left(G^{\prime}\right) \subseteq Z_{2}(G)$.

TheOREM 2.5.' Let $G$ be a 2-Engel group. Then $G$ has finitely many maximal $\mathfrak{N}_{2}$-subgroups if and only if $G / Z_{2}(G)$ is finite. In addition, each such maximal $\mathfrak{N}_{2}$-subgroup has finite index in $G . A$ 2-Engel group possessing a finite covering by $\mathfrak{N}_{2}$-subgroups does not necessarily have a second center of finite index.

Proof. Since $Z_{2}(G)$ is the margin of the variety $\mathfrak{N}_{2}$, we obtain by Lemma 2.3 that for any group $G$, the finiteness of $G / Z_{2}(G)$ implies the existence of only finitely many maximal $\mathfrak{R}_{2}$-subgroups. Assume now that $G \in \mathbb{E}_{2}$ and has finitely many maximal $\mathfrak{N}_{2}$-subgroups. Denote this set by $\mathscr{M}_{2}=\left\{H_{i}\right\}_{i=1}^{k}$. Fix $x \in G$ and let $\mathscr{A}_{x}=\left\{H_{i} \in \mathscr{M}_{2} \mid x \in H_{i}\right\}$. For any $y \in G$ we have $\langle x, y\rangle \in \mathfrak{N}_{2}$, since $\langle x, y\rangle$ is a 2-generator group in $\mathfrak{F}_{2}$. So $\langle x, y\rangle \subseteq H_{i}$ for some $i$, and $x \in\langle x, y\rangle$ implies $H_{i} \in \mathscr{A}_{x}$. Since $y$ was arbitrary, it follows that the elements in $\mathscr{A}_{x}$ form a covering of $G$. Theorem 1.2 implies that $\mathscr{B}_{x}=$ $\left\{H_{i} \in \mathscr{A}_{x} ;\left[G: H_{i}\right]<\infty\right\}$ is already a covering of $G$. Let $N_{x}=\bigcap_{H_{i} \in \mathscr{P}_{x}} H_{i}$ and $N=\bigcap_{\left|G: H_{i}\right|<\infty} H_{i}$. Then $N \subseteq \bigcap_{x \in G} N_{x}$. Consider $y \in N_{x}, g \in G$, then $y, x, g \in H_{i}$ for some $H_{i} \in \mathscr{B}_{x}$, so $[x, g, y]=1$. Thus $N_{x} \subseteq C_{G}([x, G])$, where $[x, G]=\langle[x, g] \mid g \in G\rangle$, and $N \subseteq \bigcap_{x \in G} N_{x} \subseteq$ $\bigcap_{x \in G} C_{G}([x, G])=C_{G}\left(G^{\prime}\right)$. Since $[G: N]<\infty$ and $C_{G}\left(G^{\prime}\right)=Z_{2}(G)$ by Lemma 2.4 , it follows that $\left[G: Z_{2}(G)\right]<\infty$. Another application of Lemma 2.3 shows $\left[G: H_{i}\right]<\infty$ for all $H_{i} \in \mathcal{M}_{2}$.

The group $H(3)$ constructed in the last section of this paper is a 2-Engel group which has a finite covering by $\mathfrak{N}_{2}$-subgroups, but as shown in Proposition 5.1, $Z_{2}(H(3)$ ) does not have finite index in $H(3)$.

3. Finite coverings by $n$-abelian and 2-central subgroups. In this section we discuss our second question for the cases of $n$-abelian and 2-central subgroups. The following proposition, basic in this context, will be stated without proof.

Proposition 3.1. Let \& be a group-theoretic property inherited by subgroups. A group $G$ has a finite covering by $\mathbb{E}$-subgroups if and only if $G$ has an 5 -embedded normal subgroup of finite index.

\footnotetext{
' We want to thank H. Heineken for his contribution to the proof of Theorem 2.5.
} 
Let $n$ be an integer. Two elements $x, y$ in a group $G n$-commute if $(x y)^{n}=x^{n} y^{n}$ and $(y x)^{n}=y^{n} x^{n}$ [2]. A group is $n$-abelian if any two elements in the group $n$-commute. The class of $n$-abelian groups will be denoted by $\mathfrak{A}_{n}$. R. Baer in [1] introduced the $n$-center $Z(G, n)$ of a group as the set of those elements which $n$-commute with every element in the group. In [8] it was shown that

$$
Z(G, n)=\left\{a \in G \mid(a g)^{n}=a^{n} g^{n} \forall g \in G\right\} .
$$

By replacing $Z(G)$ by $Z(G, n)$, we get the following analogue to Baer's result [12; 4.16].

THeOREM 3.2. Let $G$ be a group and $n$ a positive integer. Then:

(1) $Z(G, n)=K\left(G, \mathfrak{A}_{n}\right)$;

(2) $G$ has a finite $\mathfrak{A}_{n}$-covering if and only if $Z(G, n)$ has finite index in $G$.

Proof. We observe that (2) is a consequence of (1) and Proposition 3.1. Now consider (1). Theorem 2.3 in [8] states that $a \in Z(G, n)$ if and only if $\langle a, x\rangle$ is $n$-abelian for all $x \in G$. Thus $Z(G, n)$ is an $\mathfrak{A}_{n}$-embedded normal subgroup of $G$. Conversely, if $N$ is an $\mathfrak{I}_{n}$-embedded normal subgroup of $G$, then $\langle a, x\rangle$ is $n$-abelian for all $x \in G$ and all $a \in N$. Hence $N \subseteq Z(G, n)$. Thus $Z(G, n)$ is the unique maximal $\mathfrak{A}_{n}$-embedded normal subgroup of $G$, and $Z(G, n)=K\left(G, \mathfrak{A}_{n}\right)$.

Groups with a finite covering by normal abelian subgroups are exactly the central-by-finite 2-Engel groups [3; Theorem 2], or alternatively the central-by-finite groups with all normal closures $\left\langle x^{G}\right\rangle$ abelian. Thus our next proposition is a direct analogue of Theorem 2 in [3] in the case $n$-abelian.

PROPOSITION 3.3. A group $G$ has a finite covering by normal $n$-abelian subgroups if and only if $[G: Z(G, n)]$ is finite and $\left\langle x^{G}\right\rangle$ is $n$-abelian for all $x \in G$.

Proof. Assume $G=\bigcup_{i=1}^{k} H_{i}$, with $H_{i} \triangleleft G$ and $H_{i} \in \mathfrak{A}_{n}$. By Theorem 3.2 we have $[G: Z(G, n)]<\infty$. Let $x \in H_{i}$, then $\left\langle x^{G}\right\rangle \subseteq H_{i}$. So $\left\langle x^{G}\right\rangle \in \mathfrak{A}_{n}$ for all $x \in G$.

On the other hand, assume $[G: Z(G, n)]<\infty$, and $\left\langle x^{G}\right\rangle n$-abelian for all $x \in G$. In [8; Theorem 3.1] it has been shown that $Z(G, n)$ is the margin for the $n$-commutator word $\beta_{n}(x, y)=(x y)^{n} y^{-n} x^{-n}$. Thus by Lemma 2.3 we have that $\left\langle x^{G}\right\rangle Z(G, n)$ is an $n$-abelian normal subgroup of $G$. By Proposition 3.1 it follows that $G$ has a finite covering by $n$-abelian normal subgroups.

In [7], the characterization of groups with abelian normal closures has been extended to the case of groups with 3-abelian normal closures. Thus in the case $n=3$, a more explicit characterization can be given than the one in Proposition 3.3. This leads to the following corollary.

COROLlaRY 3.4. A group $G$ has a finite covering by normal 3-abelian subgroups if and only if $[G: Z(G, 3)]$ is finite and one of the following conditions holds:

(i) $G$ is 3-Levi, i.e. $\left[x, y^{3}\right]=[x, y]^{3}$ for all $x, y \in G$;

(ii) $G$ is 3-Bell, i.e. $\left[x, y^{3}\right]=\left[x^{3}, y\right]$ for all $x, y \in G$;

(iii) $G$ is 3-Engel and $[x, y, y]^{3}=1$ for all $x, y \in G$.

Proof. This follows immediately from Proposition 3.3 and Theorem 1 in [7], where each of the conditions (i), (ii) and (iii) has been shown to be equivalent to the statement that $\left\langle x^{G}\right\rangle \in \mathfrak{A}_{3}$ for all $x \in G$. 
Let $n$ be an integer. A group $G$ is called $n$-central if $\left[x, y^{n}\right]=1$ for all $x, y \in G$. The class of $n$-central groups will be denoted by $\beta_{n}$. It is well-known that a group is 2-central if and only if $G$ is 2-Engel and $G^{\prime}$ has exponent 2. In particular, 2-central groups are nilpotent of class 2 . We will show next that the property 2 -central is rigid.

THEOREM 3.5. Let $G$ be a group and $8_{2}$ the class of 2-central groups. Then:

(1) $L(G) \cap C_{G}\left(G^{2}\right)=K\left(G, 8_{2}\right)$;

(2) $G$ has a finite covering by $3_{2}$-subgroups if and only if $L(G) \cap C_{G}\left(G^{2}\right)$ has finite index in $G$.

As a preparation for the proof of Theorem 3.5 we need a lemma which shows in addition that the rigid $8_{2}$-kernel of a group is in general neither its $3_{2}$-margin nor a partial $3_{2}$-margin, but it contains the $3_{2}$-margin and is contained in one of the partial margins.

Lemma 3.6. Denote with $\tau(x, y)=\left[x, y^{2}\right]$ the 2-central word and let $G$ be $a$ group. Let $\tau_{1}^{*}(G)=\left\{a \in G ;\left[a x, y^{2}\right]=\left[x, y^{2}\right] \forall x, y \in G\right\}$ and $\tau_{2}^{*}(G)=\left\{a \in G ;\left[x,(a y)^{2}\right]=\right.$ $\left.\left[x, y^{2}\right] \forall x, y \in G\right\}$ be the respective partial margins, and $\tau^{*}(G)=\tau_{1}^{*}(G) \cap \tau_{2}^{*}(G)$ its $\mathrm{S}_{2}$-margin. Then:

(i) $\tau_{1}^{*}(G)=C_{G}\left(G^{2}\right)$;

(ii) $\tau_{2}^{*}(G) \subseteq Z_{2}(G)$;

(iii) $\tau^{*}(G) \subseteq L(G) \cap C_{G}\left(G^{2}\right) \subseteq \tau_{1}^{*}(G)$, where containment in both cases can be proper.

Proof. The proof of (i) is straightforward and will be omitted here. To show (ii), let $a \in \tau_{2}^{*}(G)$. Then $\left[y,(a x)^{2}\right]=\left[y, x^{2}\right]$ for all $x, y \in G$. Expansion and cancellation leads to $\left[y, \operatorname{axax}^{-1}\right]=1$, and hence $\left[y,\left[a^{-1}, x^{-1}\right]\left(a^{x^{-1}}\right)^{2}\right]=1$. Since $\left[y, b^{2}\right]=1$ for all $y \in G$ and $b \in \tau_{2}^{*}(G)$, we conclude $\left[y,\left[a^{-1}, x^{-1}\right]\right]=1$ for all $x, y \in G$, and hence $a \in Z_{2}(G)$. So $\tau_{2}^{*}(G) \subseteq Z_{2}(G)$, proving (ii).

To show (iii), we first observe that the inclusion $L(G) \cap C_{G}\left(G^{2}\right) \subseteq \tau_{1}^{*}(G)$ is obvious. To see that it can be proper, consider $S_{3}$, the symmetric group on 3 letters. We have $\tau_{1}^{*}\left(S_{3}\right)=A_{3}$ and $L\left(S_{3}\right)=1$, hence $\tau_{1}^{*}\left(S_{3}\right) \cap L\left(S_{3}\right) \neq \tau_{1}^{*}\left(S_{3}\right)$.

To prove the left hand side inclusion, we observe $\tau^{*}(G) \subseteq \tau_{2}^{*}(G)$. Thus $\tau^{*}(G) \subseteq$ $Z_{2}(G)$ by (ii). Since $Z_{2}(G) \subseteq L(G)$, we have $\tau^{*}(G) \subseteq L(G) \cap C_{G}\left(G^{2}\right)$.

To show that $\tau^{*}(G)$ can be properly contained in $L(G) \cap C_{G}\left(G^{2}\right)$, we consider the counterexample given in [6]. There, a $p$-group $H$ of nilpotency class 3 was constructed for every prime $p$, where $[H: L(H)]<\infty$, but $Z_{2}(H)$ had infinite index in $H$. For our purpose consider the case $p=2$. It can be shown that $L(H) \cap C_{H}\left(H^{2}\right)=L(H)$. Now $\tau^{*}(H) \subseteq$ $Z_{2}(H)$ by the above. Thus $\left[H: \tau^{*}(H)\right]=\infty$, hence $\tau^{*}(H) \neq L(H) \cap C_{H}\left(H^{2}\right)$.

Proof of Theorem 3.5. Observe that (2) is a consequence of (1) and Proposition 3.1. Now consider (1). Let $N$ be a $3_{2}$-embedded normal subgroup of $G$. Then $\langle x, N\rangle \in 3_{2}$ for all $x \in G$, hence $\left[a, x^{2}\right]=1$ for $x \in G$ and $a \in N$, thus $N \subseteq C_{G}\left(G^{2}\right)$. Since $\Xi_{2} \subseteq \mathbb{F}_{2}$, we have $[a, x, x]=1$ for all $x \in G$ and $a \in N$. Thus $N \subseteq L(G)$, and hence $N \subseteq L(G) \cap C_{G}\left(G^{2}\right)$.

Set $T=L(G) \cap C_{G}\left(G^{2}\right)$. We have to show that $T$ is $\}_{2}$-embedded, i.e. $\left[a x^{i},\left(b x^{j}\right)^{2}\right]=$ 1 for all $x \in G, i, j \in \mathbb{Z}$, and $a, b \in T$. By Theorem 1 in [6] we can assume that $T$, as a subgroup of $L(G)$, is an $\mathbb{E}_{2}$-embedded normal subgroup of $G$. Thus $\langle x, T\rangle \in \mathbb{F}_{2}$. This leads to the following expansion: $\left[a x^{i},\left(b x^{j}\right)^{2}\right]=\left[a,\left(b x^{j}\right)^{2}\right]^{x^{i}}\left[x, b x^{j}\right]^{2 i}=\left([x, b]^{x^{j}}\right)^{2 i}=[x, b]^{2 i}$. 
But $[x, b]^{2}=[b, x, x]$ for $b \in C_{G}\left(G^{2}\right)$ and $x \in G$. Hence we obtain $\left[a x^{i},\left(b x^{j}\right)^{2}\right]=[x, b]^{2 i}=$ $[b, x, x]^{i}=1$. Thus $T$ is a $B_{2}$-embedded normal subgroup and we conclude $K\left(G, B_{2}\right)=$ $L(G) \cap C_{G}\left(G^{2}\right)$.

The properties shown to be rigid so far are all varieties defined by a 2-variable law. Specifically, in the case of the varieties abelian and $n$-abelian, defined by symmetric laws, the rigid kernel turned out to be the respective margin. As shown in [6], in the case of $\mathfrak{F}_{2}$ we have $K\left(G, \mathfrak{F}_{2}\right)=L(G)$, which is a partial margin for $\mathbb{F}_{2}$. In the case of 2-central we have yet another situation. As shown in Theorem 3.5 and Lemma 3.6, $K\left(G, B_{2}\right)$ can strictly contain the $B_{2}$-margin and can be properly contained in one of its partial margins. The outcome seems to depend very much on the structure of the individual law in question. In the next section we consider a variety defined by a 3-variable law.

4. Finite coverings by subgroups of class 2 . In this section we focus on the property nilpotency of class 2 in the context of our second question. The main result is that $\mathfrak{N}_{2}$ is not a rigid property. This does not preclude the existence of a characteristic subgroup in every group whose finite index characterizes groups having a finite $\mathfrak{N}_{2}$-covering. Our result only says that such a characteristic subgroup is not the rigid kernel for the property $\mathfrak{N}_{2}$. Here is the main result of this section.

TheOREM 4.1. (i) A group $G$ can have more than one maximal $\mathfrak{N}_{2}$-embedded normal subgroup. (ii) If $G$ is 3-torsionfree, then $L(G)$ is the unique maximal $\mathfrak{N}_{2}$-embedded normal subgroup of $G$.

Proof. An example of a finite group having more than one maximal $\mathfrak{N}_{2}$-embedded normal subgroup is $B=B(3,3)$, the 3-generator Burnside group of exponent 3 . We have $B \in \mathfrak{N}_{3}, B^{\prime}=Z_{2}(B)$. It can easily be shown that $\left\langle g, Z_{2}(B)\right\rangle$ is a maximal $\mathfrak{N}_{2}$-embedded normal subgroup of $B$ for every $g \in B \backslash B^{\prime}$.

The group $H(3)$ constructed in the next section possesses maximal $\mathfrak{N}_{2}$-embedded normal subgroups of finite as well as of infinite index, as shown in Proposition 5.1.

(ii) We observe that by [6; Theorem 1] we have $\langle g, L(G)\rangle \in \mathbb{E}_{2}$ for all $g \in G$. Now assume that $G$ has no elements of order 3 . Then $\langle g, L(G)\rangle \in \mathfrak{N}_{2}$. Thus $L(G)$ is an $\mathfrak{N}_{2}$-embedded normal subgroup of $G$.

Conversely, assume $N \triangleleft G$ and $\langle g, N\rangle \in \mathfrak{N}_{2}$ for all $g \in G$. Then $[a, g, g]=1$ for $a \in N$ and all $g \in G$, hence $N \subseteq L(G)$. If $G$ is 3-torsionfree, then $L(G) \in \mathfrak{N}_{2}$, and thus $L(G)$ is the unique maximal $\mathfrak{N}_{2}$-embedded normal subgroup of $G$.

A reformulation of Theorem 4.1.ii together with Proposition 3.1 gives us the following corollary.

Corollary 4.2. Let $G$ be a 3-torsionfree group. Then $G$ has a finite covering by $\mathfrak{N}_{2}$-subgroups if and only if $L(G)$ has finite index in $G$.

The last result of this section shows that groups having a finite covering by $\mathfrak{N}_{2}$-subgroups can be characterized by the existence of a suitably embedded normal subgroup of finite index, though this subgroup need not be characteristic.

THEOREM 4.3. A group $G$ has a finite covering by $\mathfrak{N}_{2}$-subgroups if and only if $G$ has a normal subgroup $N$ of finite index with $N \subseteq L(G)$ and $N^{\prime} \subseteq Z(G)$. 
Proof. Assume $G=\bigcup_{i=1}^{k} H_{i}, H_{i} \in \Re_{2}$. By Theorem 1.2 we may assume $\left[G: H_{i}\right]<\infty$. Then $N=\operatorname{cor}\left(\bigcap_{i=1}^{k} H_{i}\right)$ is a normal subgroup of finite index. Let $n, n^{\prime} \in N, g \in G$. Then $g \in H_{i}$ for some $i$. Thus $[n, g, g]=1$ and $\left[n, n^{\prime}, g\right]=1$. Hence $N \subseteq L(G)$ and $N^{\prime} \subseteq Z(G)$.

Conversely, assume $N \triangleleft G, N \subseteq L(G)$ and $N^{\prime} \subseteq Z(G)$. By [6; Theorem 1] we have $\langle g, N\rangle \in \mathbb{E}_{2}$ for all $g \in G$, since $N \subseteq L(G)$. We need to show $\langle g, N\rangle \in \mathfrak{N}_{2}$, or equivalently $\left[g^{i_{1}} n_{1}, g^{i_{2}} n_{2}, g^{i_{3}} n_{3}\right]=1$ for $g \in G, i_{1}, i_{2}, i_{3} \in \mathbb{Z}$, and $n_{1}, n_{2}, n_{3} \in N$. Let $x \in\langle g, N\rangle$ and $n, n^{\prime} \in N$. Since $\langle g, N\rangle \in \mathbb{E}_{2}$ and $N^{\prime} \subseteq Z(G)$, we obtain

$$
\left[x, n, n^{\prime}\right]=\left[n, x, n^{\prime}\right]^{-1}=\left[n, n^{\prime}, x\right]=1 .
$$

Observing $\left[g^{i_{1}} n_{1}, g^{i_{2}} n_{2}, g^{i_{3}} n_{3}\right] \in Z(\langle g, N\rangle)$, we may expand linearly and obtain together with the above that $\left[g^{i_{1}} n_{1}, g^{i_{2}} n_{2}, g^{i_{3}} n_{3}\right]=1$. Thus $\langle g, N\rangle \in \mathfrak{N}_{2}$ for all $g \in G$. Hence $N$ is an $\mathfrak{N}_{2}$-embedded normal subgroup of $G$. If in addition $[G: N]<\infty$, it follows by Proposition 3.1 that $G$ has a finite covering by $\mathfrak{R}_{2}$-subgroups.

5. A counterexample. In this section a 3-group $H(3)$ is constructed whose relevant properties are stated in Proposition 5.1. The construction of $H(3)$ follows usual practice, and in this case it is similar to the one given in [6]. Starting from a group isomorphic to the commutator subgroup of $H(3)$, we will reach $H(3)$ by three split extensions.

Construction of the Counterexample. Let $E_{3}$ denote an elementary abelian 3-group of countable rank. Set $V=\left\langle v_{1}, v_{2}, \ldots\right\rangle, W=\left\langle w_{1}, w_{2}, \ldots\right\rangle$ and $Z=\left\langle z_{1}, z_{2}, \ldots\right\rangle$ with $V \cong W \cong Z \cong E_{3}$. Define $X=\langle u\rangle \times V \times W \times Z$ with $\langle u\rangle \cong C_{3}$.

Let $A=[X]\langle a\rangle$, the semidirect product of $X$ with a cyclic group $\langle a\rangle \cong C_{3}$. The automorphism induced by $a$ on $X$ has order 3 , where the action of $a$ on the generators of $X$ is given as follows:

$$
[u, a]=\left[v_{i}, a\right]=\left[z_{i}, a\right]=1, \quad\left[w_{i}, a\right]=z_{i}, \quad i=1,2, \ldots
$$

The defining relations of $A$ are those of $X,(1)$, and $a^{3}=1$.

Similarly, let $B=[A]\langle b\rangle$ with $\langle b\rangle \cong C_{3}$, where $b$ induces an automorphism of order 3 on $A$. The action of $b$ on the generators of $A$ is given as follows:

$$
[a, b]=u, \quad[u, b]=\left[w_{i}, a\right]=\left[z_{i}, b\right]=1, \quad\left[v_{i}, b\right]=z_{i}^{-1}, \quad i=1,2, \ldots
$$

The defining relations of $B$ are those of $A,(2)$, and $b^{3}=1$.

For the last extension we set $C=\left\langle c_{1}, c_{2}, \ldots\right\rangle \cong E_{3}$. Let $H(3)=[B] C$. The elements of $C$ induce automorphisms of order 3 on $B$. The action of the generators of $C$ on the generators of $B$ is given as follows:

$$
\left.\begin{array}{l}
{\left[a, c_{i}\right]=v_{i}, \quad\left[b, c_{i}\right]=w_{i}, \quad\left[u, c_{i}\right]=z_{i}, \quad i=1,2, \ldots} \\
{\left[v_{i}, c_{j}\right]=\left[w_{i}, c_{j}\right]=\left[z_{i}, c_{j}\right]=1, \quad i, j=1,2, \ldots}
\end{array}\right\}
$$

The defining relations of $H(3)$ are those of $B,(3)$, and those of $C$. This concludes the construction of $H(3)$.

For the notation in Proposition 5.1 we refer to that used in the construction of $H(3)$. 
Proposition 5.1. Let $H=H(3)$ be the group constructed above. Then:

(i) $H=\langle a, b, C\rangle$ and $c(H)=3$, precisely;

(ii) $H$ is a 2-Engel group and $\exp H=3$;

(iii) $H^{\prime}=Z_{2}(H)$ and $\left[H: Z_{2}(H)\right]$ is infinite;

(iv) $H$ has maximal $\mathfrak{P}_{2}$-embedded normal subgroups of finite and infinite index. In particular, $H$ has infinitely many maximal $\mathfrak{N}_{2}$-embedded normal subgroups of infinite index;

(v) $H$ has maximal $\mathfrak{N}_{2}$-subgroups of finite and infinite index. In particular, $H$ has infinitely many maximal $\mathfrak{N}_{2}$-subgroups of infinite index;

(vi) $H$ has a finite covering by $\mathfrak{N}_{2}$-subgroups.

Proof. The verification of (i) is straightforward and will be omitted here.

(ii) Let $h=a^{\alpha} b^{\beta} c x, \alpha, \beta \in \mathbb{Z}, c \in C, x \in X$. By (i) we can expand linearly and, using the relations of $H$, we obtain $\left[c_{i}, h, h\right]=\left(\left[v_{i}, b\right]\left[w_{i}, a\right]\right)^{-\alpha \beta}=\left(z_{i}^{-1} z_{i}\right)^{-\alpha \beta}=1$. Hence $C \subseteq L(H)$. Every $c \in C$ can be written as $c=\prod_{i \in I_{c}} c_{i}^{\rho_{i}}$, where $\rho_{i} \in \mathbb{Z}$ and $I_{c}$ is a finite index set. We write $v=\prod_{i \in I_{c}} v_{i}^{\rho_{i}}, w=\prod_{i \in I_{c}} w_{i}^{\rho_{i}}$, and $z=\prod_{i \in I_{c}} z_{i}^{\rho_{i}}$. Using the relations of $H$, we obtain with this notation

$$
[a, h, h]=[u, c]^{\beta}[a, c, b]=(z[v, b])^{\beta}=\left(z z^{-1}\right)^{\beta}=1,
$$

and similarly $[b, h, h]=1$. We conclude $a, b \in L(H)$, and hence $H=\langle a, b, C\rangle \subseteq L(H)$. Thus $H \in \mathbb{E}_{2}$. It follows that $\exp H=3$, since $H$ is generated by elements of order 3 .

(iii) By (i) we obviously have $H^{\prime} \subseteq Z_{2}(H)$. Let $y=a^{\alpha} b^{\beta} c y^{\prime} \in Z_{2}(H)$, where $\alpha, \beta \in \mathbb{Z}$, $c \in C$, and $y^{\prime} \in H^{\prime}$. Then $[y, g, h]=1$ for all $g, h \in H$. By linear expansion this leads to

$$
1=[a, g, h]^{\alpha}[b, g, h]^{\beta}[c, g, h] .
$$

Setting $h=c_{1}$ and $g=b$ or $g=a$ in (4), we obtain $\left[a, b, c_{1}\right]^{\alpha}=1$ or $\left[b, a, c_{1}\right]^{\beta}=1$, respectively. Hence $\alpha \equiv \beta \equiv 0 \bmod 3$. By setting $g=a$ and $h=b,(4)$ becomes $[c, a, b]=$ 1. It remains to be shown that $c=1$. Linear expansion together with the relations of $H$ yield $1=[c, a, b]=\prod_{i \in I_{c}}\left[c_{i}, a, b\right]^{\rho_{i}}=\prod_{i \in I_{c}} z_{i}^{\rho_{i}}=z$. Now $z=1$ implies $\rho_{i} \equiv 0 \bmod 3$. But then $c=\prod_{i \in I_{c}} c_{i}^{\rho_{i}}=1$. Hence $y=y^{\prime} \in H^{\prime}$, and thus $Z_{2}(H) \subseteq H^{\prime}$. It can easily be seen that $H^{\prime}=X$. Since $[H: X]=\infty$, it follows that $\left[H: Z_{2}(H)\right]=\infty$.

(iv) Consider $B_{j}=\left\langle b c_{j}, Z_{2}(H)\right\rangle, j=1,2, \ldots$ We observe that $\left\langle g, b c_{j}\right\rangle \in \mathfrak{N}_{2}$ for all $g \in H$ as a 2-generator group in $\mathfrak{E}_{2}$. Thus, by Lemma 2.3, $\left\langle g, B_{j}\right\rangle \in \mathfrak{J}_{2}$ for all $g \in H$. Now $B_{j} \triangleleft H$ by (iii). Hence $B_{j}$ is an $\mathfrak{N}_{2}$-embedded normal subgroup of $H$. Assume $B_{j}$ is not maximal in that respect. Then there exists $g \in H \backslash B_{j}$, such that $\tilde{B}_{j}=\left\langle g, B_{j}\right\rangle$ is $\mathfrak{N}_{2^{-}}$ embedded, i.e. $\left\langle y, \tilde{B}_{j}\right\rangle \in \mathfrak{N}_{2}$ for all $y \in H$. Without loss of generality let $g=a^{\alpha} b^{\beta} c$, $\alpha, \beta \in \mathbb{Z}$, and $c \in C$. By linear expansion we obtain

$$
\left[g, b c_{j}, y\right]=[a, b, y]^{\alpha}\left[a, c_{j}, y\right]^{\alpha}\left[b, c_{j}, y\right]^{\beta}[c, b, y] .
$$

If $\alpha \neq 0 \bmod 3$, let $y=b$, and hence $\left[g, b c_{j}, b\right]=z_{j}^{-\alpha} \neq 1$. Similarly, if $\alpha \equiv \beta \equiv 0 \bmod 3$ and $c \neq 1$, let $y=a$, and hence $\left[g, b c_{j}, a\right]=z^{-1} \neq 1$. Thus we can assume $g=b^{\beta} . c$, $\beta \neq 0 \bmod 3$. Setting $y=a$, the relations of $H$ and (5) yield $\left[g, b c_{j}, a\right]=z_{j}^{\beta} z^{-1}$. If $\left[g, b c_{j}, a\right]=1$, we obtain $z=z_{j}^{\beta}$, and hence $c=c_{j}^{\beta}$. This implies $g=b^{\beta} c_{j}^{\beta} \in B_{j}$. We 
conclude that $B_{j}$ is a maximal $\mathfrak{N}_{2}$-embedded normal subgroup of $H$, and obviously $\left[H: B_{j}\right]=\infty$. Thus $\left\{B_{j}\right\}_{j=1}^{\infty}$ is an infinite set of $\mathfrak{N}_{2}$-embedded normal subgroups of $H$, each having infinite index.

Consider $N=C Z_{2}(H)$. Then $N \in \mathfrak{P}_{2}$ and $N \triangleleft H$ by Lemma 2.3 and (iii), and obviously $[H: N]=3^{2}$. To prove $\langle g, N\rangle \in \mathfrak{N}_{2}$ for all $g \in H$, we need to show $\left[x_{1}, x_{2}, x_{3}\right]=1$ for all $x_{1}, x_{2}, x_{3} \in\langle g, N\rangle$. Let $x_{j}=g^{i} n_{j}, i_{j} \in \mathbb{Z}$ and $n_{j} \in N, j=1,2,3$. Without loss of generality we can assume $n_{j} \in C$. Then, observing (ii) and using linear expansion, we obtain $\left[x_{1}, x_{2}, x_{3}\right]=1$. Thus $\langle g, N\rangle \in \mathfrak{N}_{2}$ for all $g \in H$. If $g \notin N$, then $\langle g, N\rangle$ is a maximal subgroup of $H$. By (i), it follows that $N$ is a maximal $\mathfrak{N}_{2}$-embedded subgroup of finite index.

(v) From the preceding remark in (iv) we immediately see that $\left\langle g, C Z_{2}(H)\right\rangle$ is a maximal $\mathfrak{N}_{2}$-subgroup of $H$ of finite index, provided $g \notin C Z_{2}(H)$. Consider $M_{j}=\left\langle a, B_{j}\right\rangle$, $j=1,2, \ldots$ By (iv), we obviously have $M_{j} \in \mathfrak{N}_{2}$. We have to show that $M_{j}$ is a maximal $\mathfrak{H}_{2}$-subgroup, or equivalently $\left\langle g, M_{j}\right\rangle \in \mathfrak{N}_{2}$ implies $g \in M_{j}$. Let $g=a^{\alpha} b^{\beta} c y^{\prime}, \alpha, \beta \in \mathbb{Z}$, $c \in C, y^{\prime} \in H^{\prime}$, and assume $\left\langle g, M_{j}\right\rangle \in \mathfrak{M}_{2}$. Using the relations of $H$, we obtain by linear expansion $1=\left[a, b c_{j}, g\right]=z . z_{j}^{-\beta}$. Thus $z_{j}^{\beta}=z$, and hence $c=c_{j}^{\beta}$. But then $g \in M_{j}$. As in (iv) we conclude that $\left\{M_{j}\right\}_{j=1}^{\infty}$ is an infinite set of maximal $\mathfrak{R}_{2}$-subgroups of infinite index.

(vi) By (iv), we have that $C Z_{2}(H)$ is an $\mathfrak{N}_{2}$-embedded normal subgroup of finite index in $H$. Thus Proposition 3.1 implies that $H$ has a finite covering by $\Re_{2}$-subgroups.

\section{REFERENCES}

1. R. Baer, Endlichkeitskriterien für Kommutatorgruppen, Math. Annalen 124 (1952), $166-177$.

2. R. Baer, Factorization of $n$-soluble and $n$-nilpotent groups, Proc. Amer. Math. Soc. 4 (1953), 15-26.

3. M. A. Brodie, R. F. Chamberlain and L. C. Kappe, Finite coverings by normal subgroups, Proc. Amer. Math. Soc. 104 (1988), 669-674.

4. A. Dietrich, Durch Einbettung beschriebene charakteristische Untergruppen, Diplomarbeit, Würzburg, 1990.

5. P. Hall, Verbal and Marginal Subgroups, J. Reine Angew. Math. 182 (1940), 156-157. 141-150.

6. L. C. Kappe, Finite coverings by 2-Engel groups, Bull. Austral. Math. Soc. 38 (1988),

7. L. C. Kappe and R. F. Morse, Groups with 3-abelian normal closures, Arch. Math. 51 (1988), 104-110.

8. L. C. Kappe and M. L. Newell, On the $n$-centre of a group, Groups St. Andrews 1989, LMS Lecture Notes 160 (1991), 339-352. 227-242.

9. B. H. Neumann, Groups covered by finitely many cosets, Publ. Math. Debrecen 3 (1954),

10. B. H. Neumann, Groups covered by permutable subsets, J. London Math. Soc. 29 (1954), 236-248.

11. B. H. Neumann, A problem of Paul Erdös on groups, J. Austral. Math. Soc. 21 A (1976), 467-472.

12. D. J. S. Robinson, Finiteness conditions and generalized soluble groups, Part I, (Springer-Verlag 1972).

Adirondack Community College

QueENSBURY, NY 12804-1498

USA
SUNY AT BINGHAMTON

Department of Mathematical Sciences

BINGHAMTON, NY 13902-6000

USA 